\title{
Dynamics of Schistosoma Mansoni Development in The Intermediate Host, Biomphalaria Glabrata, A Freshwater Snail
}

\author{
Yukiteru Ouji ( $D$ oujix@naramed-u.ac.jp ) \\ Nara Medical University \\ Masayasu Misu \\ Nara Medical University \\ Tomotaka Kitamura \\ Nara Medical University \\ Megumi Hamasaki \\ Nagasaki University \\ Shinjiro Hamano \\ Nagasaki University \\ Masahide Yoshikawa \\ Nara Medical University
}

\section{Research}

Keywords: Schistosomiasis, mansoni, immunohistochemical, hepatopancreas, Biomphalaria glabrata

Posted Date: December 23rd, 2020

DOI: https://doi.org/10.21203/rs.3.rs-133333/v1

License: () (1) This work is licensed under a Creative Commons Attribution 4.0 International License. Read Full License 


\section{Abstract}

\section{Background}

Schistosomiasis is one of the most significant and prevalent waterborne parasitic diseases. Even though many studies have been reported about schistosomiasis, the dynamics of schistosome in intermediate host snails is little known. In the present study, the dynamics of Schistosoma larvae in infected snails was histologically investigated.

\section{Methods}

To examine the localization of Schistosoma mansoni (S. mansoni) parasites in the snails, Biomphalaria glabrata snails infected with miracidia were harvested and examined by stereoscopic observation. Then, frozen sections were prepared and stained with H\&E. Furthermore, immunohistochemical detection of parasites was performed using anti-S. mansoni antibody, and their localization in the snails was analyzed.

\section{Results}

Snails infected with S. mansoni miracidia were harvested at 10 and 56 days post-infection (DPI) and analyzed. In the stereoscopic observations, white spots were observed at $56 \mathrm{DPI}$, while no spots were observed at $10 \mathrm{DPI}$. However, histological investigations visualized the larvae specifically in the head-foot area of the snail at $10 \mathrm{DPI}$. Further, it was observed that the larvae relocated to the hepatopancreas and ovotestis areas at $56 \mathrm{DPI}$.

\section{Conclusions}

The present study revealed the dynamics of Schistosoma larvae in intermediate snails, shown as the differential localization of $S$. mansoni larvae at early and late infection stages.

\section{Background}

Schistosomiasis is one of the most significant and prevalent waterborne parasitic diseases, as it affects more than 240 million people worldwide (WHO 2020) [1, 2]. Six Schistosoma species (Schistosoma haematobium, S. mansoni, S. japonicum, S.intercalatum, S. guineensis, S. mekongi) are pathogenic in humans and freshwater snails are known to be their intermediate hosts [3, 4]. Following excretion of schistosome eggs from an infected human or animal, miracidia hatch from the eggs and infect intermediate host snails, with developing into cercariae in the snails [5, 6]. After cercariae are released from the snails, they percutaneously infect humans and animals, then become schistosomulae, migrate into the portal vein via the bloodstream, and mature into adult worms [5, 7]. Combinations of male and female adult worms lay eggs in intestinal or pelvic veins, and the schistosome lifecycle is repeated [8]. 
A large number of studies have been reported about schistosomiasis, including case reports and developments of new drugs and vaccination methods [9-14]. On the other hand, few studies that focused on intermediate hosts have been presented [15-17]. Notably, the dynamics of schistosome in intermediate host snails is little known, though it has been well known that the infection begins with miracidia invasion and ends with explosively increased production of cercariae. Since Schistosoma cercariae infect the final host, it is crucial to suppress outbreak production of cercariae to control the prevalence of schistosomiasis.

In the present study, the dynamics of Schistosoma larvae in infected snails was histologically visualized and we revealed that the larvae initially invade the surface of the head-foot area of the snail and localize there in the early period of their development, then relocate to the hepatopancreas and ovotestis areas in the late period of the infection. Thus, it is elucidated that the process of Schistosoma larvae growth and development is related to the selection of preferential locations inside intermediate host snails.

\section{Methods}

\section{Ethical statement}

All of the present experiments using Schistosoma mansoni (S. mansoni) parasites, Biomphalaria glabrata (B. glabrata) snails, and mice were approved by the Institutional Animal Research Committee of Nara Medical University $(\# 12813,12776)$, and performed according to Japanese statutes regarding Humane Treatment and Management of Animals.

\section{Animals and parasites}

Female ICR mice (eight weeks old) were purchased from Japan SCL (Hamamatsu, Japan). A strain of $S$. mansoni (NIH-Sm-PR-1) was maintained at the animal facilities of Nara Medical University by passage through albino B. glabrata snails (Newton's NIH Puerto Rican/Brazilian M-line strain) and ICR mice [18]. Mice were subcutaneously infected with $250 \mathrm{~S}$. mansoni cercariae, then the livers were harvested eight weeks after initiation of infection. Parasite eggs were separated from the livers and collected following treatments with actinase and collagenase. Miracidia were then hatched from the eggs in sterile water and used to infect $B$. glabrata snails (5-mm shell size). Each snail was infected with five miracidia of $S$. mansoni. They were placed in 24-well plates containing tap water for 24 hours at $28^{\circ} \mathrm{C}$, then transferred into a 15-L aquarium. The release of cercariae was observed by illumination with an LED lamp from four weeks up to 12 weeks after infection.

\section{Post-infection histological analysis of $B$. glabrata in snails}

To examine the localization of the parasites in the snails, $B$. glabrata snails infected with miracidia were harvested at 10 and 56 days post-infection (DPI) (Fig. 1). After removing the shell, each snail was fixed with $4 \%$ PFA for two days at $4^{\circ} \mathrm{C}$, followed by $20 \%$ sucrose in PBS for 12 hours. Next, the specimens were embedded in an OTC compound, then sections (10 $\mu \mathrm{m}$ thick) were prepared using a cryostat (Thermo) 
and stained with hematoxylin-eosin (H\&E) using a standard protocol. Immunohistochemical detection of S. mansoni parasites was performed in each of 10 sections and their localization in the snails was analyzed. For immunohistochemistry, the sections were permeabilized with $0.2 \%$ Triton X-100 in PBS (TPBS) containing $1 \%$ BSA, followed by blocking with $5 \%$ normal goat serum, then treated with a mouse anti-S. mansoni monoclonal antibody (BGN/1A7, dilution 1:100, Novus Biologicals). The following incubation at $4^{\circ} \mathrm{C}$ overnight and washing three times with TPBS, an AlexaFluor 546 conjugated goat antimouse secondary antibody (dilution 1:200, Molecular Probes, Invitrogen) was used to detect primary antibodies. All nuclei were stained with DAPI (Dojin), followed by washing three times with TPBS. Fluorescence was visualized using a fluorescent microscopy system (Zeiss Axiovert 200).

\section{Results}

\section{Localization of $S$. mansoni larvae in infected-B. glabrata snails}

Snails infected with S. mansoni miracidia were harvested at 10 and 56 DPI and analyzed. At 10 DPI (early infection), no gross abnormalities were observed in the infected snails as compared with uninfected snails (Fig. 2A). At 56 DPI (late infection), white spots were noted in the infected snails (Fig. 2B, 2F-2J), whereas those were not observed in uninfected snails at any time (Fig. 2A-2E), indicating that the spots were formed where $S$. mansoni sporocysts or cercariae existed. The snails were fixed and sliced, then the sections were subjected to H\&E staining. In sections from the head-foot (HF) area at $10 \mathrm{DPI}$, deep purple colored structures were detected, in which the presence of $S$. mansoni larvae was suspected (Fig. 3A-3C, 3E, 3F), while it was not detected in the hepatopancreas (HP) area (Fig. 3D). At 56 DPI, S. mansoni larvae were detected in the deep purple colored structures in the sections of the HP area (Fig. 3G), while more precise observations revealed their distribution in other organs, including the ctenidium (Ct), and ovotestis (OT) (Fig. 3I-3K), but not in HF area (Fig. 3H).

After serial sections were stained by H\&E (Fig. 4A, 4B, 4N, 40), immunohistochemical analysis was conducted using the anti-S. mansoni antibody that reacts to both $S$. mansoni miracidia and cercariae (Supplementary Fig. S1). Figures A, B, N and O in Figure 4 are same respectively to Figures A, B, G and J in Figure 3. S. mansoni larvae were immunohistochemically confirmed to be present in the HF area in the early period of their development (10 DPI) (Fig. 4A-4M). However, they were predominantly present in the $\mathrm{HP}$ areas in the late period of their development (56 DPI) (Fig. 4N-4R).

\section{Mapping of S. mansoni larvae in B. glabrata snails}

We attempted to produce a map showing where S. mansoni larvae existed in B. glabrata snails (Fig. 5A). Immunohistochemical analysis of serial sections of $S$. mansoni-infected B. glabrata snails at $10 \mathrm{DPI}$ showed larvae in 30 out of 45 sections from the HF area (66\%) (Fig. 5B), while those were observed in only 7 of 45 sections from other areas (15\%), such as $\mathrm{Hr}$ and Ct. At $56 \mathrm{DPI}$, immunopositivity for $S$. mansoni larvae was observed in all 15 successive specimens from the HP and OT areas, which was in contrast to sparse distribution in the other areas, indicating accumulation of $S$. mansoni larvae in the HP + OT (HPOT) area (Fig. 5C). These findings indicate that S. mansoni larvae become located in the HF area 
during the early infection period, then move to the HPOT area by the time of late infection, and suggest the presumable location-specific assistance.

\section{Discussion}

Schistosome infects intermediate host snails with miracidia, then miracidia become cercariae by approximately four weeks $[5,19]$. Although it is known that the intra-snail development process includes mother sporocysts, daughter sporocysts, and cercariae $[4,7]$, their distributions in snails have not been analyzed in detail. In the present study, localization of $S$. mansoni larvae during the early (10 DPI) and late (56 DPI) development periods in intermediate host $B$. glabrata snails was investigated, and $t$ the distribution of $S$. mansoni larvae was clearly demonstrated to be different between those early and late periods, as the predominant location at $10 \mathrm{DPI}$ was the head-foot (HF) area, while the hepatopancreas (HP) and ovotestis (OT) areas were predominant at $56 \mathrm{DPI}$. However, the larval developmental stage was not determined due to the limitation that anti-S. mansoni monoclonal antibody used in the present study recognizes both $S$. mansoni miracidia and cercariae (Supplementary Fig. S1) but does not discriminate each stages in the snails. Bahia et al. reported that the S. mansoni protein kinase C1 (SmPKC1) is differentially expressed during the lifecycle of this parasite (adult worm, miracidium, sporocyst, cercaria, schistosomula) [20], and Fernandes et al. found that expression patterns of the $S$ mansoni venom allergen-like protein (SmVAL) family differed among the germ ball, cercaria, and schistosomula [21]. We consider that immunohistological analysis of SmPKC1 and SmVAL expression patterns would make it possible to identify the developmental stage of larvae found at 10 and $56 \mathrm{DPI}$.

Schistosome infects only certain species of intermediate host snails, while $B$. glabrata is known to be the host snail for S. mansoni [6, 7]. The albino B. glabrata snails (Newton's NIH Puerto Rican/Brazilian M-line strain) used in the present study have been reported to produce $S$. mansoni cercariae [18], which was confirmed by our findings. Some strains of $B$. glabrata, such as the NMRI and M line, are also susceptible to the infection by $S$. mansoni [22, 23], while resistant strains such as BS-90 also allow infection with $S$. mansoni miracidia, though it does not lead to production of cercariae [24,25]. Snails have innate immune defense systems that can specifically or non-specifically target schistosomes for destruction [26, 27]. Even following successful infection, miracidia and sporocysts are exposed to humoral factors, and attacked by motile hemocytes in the hemolymph and other tissues of the snail [28], resulting in production failure of cercariae. Therefore, an immunohistological investigation of larval distribution using resistant snails such as BS-90 may provide temporal and spatial information regarding the arrest of larval development. Indeed, larval encapsulation and several factors including reactive oxygen species[29] and other resistance-associated factors [30] have been noted as possible mechanisms by which the development of larvae fails to proceed.

The present results demonstrated changes in larval locations inside snails between early and late infection, strongly suggesting that the environment favoring Schistosoma larvae differs depending on the developmental stage. Several methods for culturing Schistosoma larvae in vitro have been reported [31, 32]. The Bge cell line, established from 4- to 5-day-old embryos of an albino B. glabrata snail, has been 
utilized to support the development and growth of Schistosoma spp [33]. larvae in vitro. According to the report by Yoshino et al., Bge cells supported the induction of sporocysts but failed to produce cercariae [34]. Furthermore, Ivanchenko et al. observed the generation of daughter sporocysts, whereas cercariae production was scarcely observed in the cultures with Bge cells [35]. Considering previously reported information about culture systems, the use of Bge cells alone seems to be inadequate to achieve complete production of sporocysts and cercariae. Identification of environmental factors required for the development of those larvae would make it possible to grow them in vitro.

\section{Conclusion}

The present study revealed differential localization of $S$. mansoni larvae at early and late infection stages in intermediate snails. Our findings may lead to the development of novel methods for in vitro larvae cultivation using larval stage-specific environmental factors.

\section{Abbreviations}

S. mansoni. Schistosoma mansoni

B. glabrata: Biomphalaria glabrata

DPI: days post-infection

H\&E: hematoxylin-eosin

TPBS: $0.2 \%$ Triton $\mathrm{X}-100$ in PBS

HF: head-foot

HP: hepatopancreas

Ct: ctenidium

OT: ovotestis

Hr: heart

HPOT: hepatopancreas and ovotestis

SmPKC1: S. mansoni protein kinase C1

SmVAL: S mansoni venom allergen-like protein

\section{Declarations}


We thank Mrs. S. Shimada for the technical assistance.

\section{Funding}

This work was supported by the Joint Usage / Research Center on Tropical Disease, Institute of Tropical Medicine, Nagasaki University (2019-Ippan-2, 2020-Ippan-20).

Availability of data and materials

All data generated or analyzed during this study are included in this article and its supplementary information files.

Author information

Affiliations

Department of Pathogen, Infection and Immunity, Nara Medical University, Kashihara, Nara, Japan

Yukiteru Ouji, Masayasu Misu, Tomotaka Kitamura, Masahide Yoshikawa

Department of Parasitology, Institute of Tropical Medicine (NEKKEN), Nagasaki University, Nagasaki, Japan

Megumi Hamasaki, Shinjiro Hamano

The Joint Usage/Research Center on Tropical Disease, Institute of Tropical Medicine (NEKKEN), Nagasaki University, Nagasaki, Japan

Megumi Hamasaki, Shinjiro Hamano

Program for Nurturing Global Leaders in Tropical and Emerging Communicable Diseases, Graduate School of Biomedical Sciences, Nagasaki University, Nagasaki, Japan

Shinjiro Hamano

Nagasaki University, Kenya Research Station, NUITM-KEMRI Project, Nairobi, Kenya

Shinjiro Hamano

\section{Contributions}

$\mathrm{YO}, \mathrm{SH}, \mathrm{MY}$ designed the experiments. $\mathrm{YO}, \mathrm{MH}, \mathrm{MM}$ performed the experiments. $\mathrm{YO}, \mathrm{MH}, \mathrm{SH}, \mathrm{MY}$ collected and analyzed the data. MM, MH, TK contributed reagents/materials/analysis. $\mathrm{YO}, \mathrm{SH}, \mathrm{MY}$ wrote the paper. All authors read and approved the final version of the manuscript. 
Correspondence to Yukiteru Ouji.

\section{Ethics declarations}

\section{Ethics approval and consent to participate}

All of the present experiments were approved by the Institutional Animal Research Committee of Nara Medical University $(\# 12813,12776)$, and performed according to Japanese statutes regarding Humane Treatment and Management of Animals.

\section{Consent for publication}

Not applicable.

\section{Competing interests}

The authors declare that they have no competing interests.

\section{References}

1. WHO Fact Sheet on Schistosomiasis (2020) https://www.who.int/healthtopics/schistosomiasis\#tab=tab_1.

2. Colley DG, Bustinduy AL, Secor WE, King CH. Human schistosomiasis. Lancet. 2014;383 9936:225364; doi: 10.1016/S0140-6736(13)61949-2. https://www.ncbi.nlm.nih.gov/pubmed/24698483.

3. Steinmann P, Keiser J, Bos R, Tanner M, Utzinger J. Schistosomiasis and water resources development: systematic review, meta-analysis, and estimates of people at risk. Lancet Infect Dis. 2006;6 7:411-25; doi: 10.1016/S1473-3099(06)70521-7. https://www.ncbi.nlm.nih.gov/pubmed/16790382.

4. McManus DP, Dunne DW, Sacko M, Utzinger J, Vennervald BJ, Zhou XN. Schistosomiasis. Nat Rev Dis Primers. 2018;4 1:13; doi: 10.1038/s41572-018-0013-8. https://www.ncbi.nlm.nih.gov/pubmed/30093684.

5. Gryseels B, Polman K, Clerinx J, Kestens L. Human schistosomiasis. Lancet. 2006;368 9541:1106-18; doi: 10.1016/S0140-6736(06)69440-3. https://www.ncbi.nlm.nih.gov/pubmed/16997665.

6. Gurarie D, Lo NC, Ndeffo-Mbah ML, Durham DP, King CH. The human-snail transmission environment shapes long term schistosomiasis control outcomes: Implications for improving the accuracy of predictive modeling. PLoS neglected tropical diseases. 2018;12 5:e0006514; doi: 10.1371/journal.pntd.0006514. https://www.ncbi.nlm.nih.gov/pubmed/29782500.

7. Nation CS, Da'dara AA, Marchant JK, Skelly PJ. Schistosome migration in the definitive host. PLoS neglected tropical diseases. 2020;14 4:e0007951; doi: 10.1371/journal.pntd.0007951. https://www.ncbi.nlm.nih.gov/pubmed/32240157. 
8. Nelwan ML. Schistosomiasis: Life Cycle, Diagnosis, and Control. Curr Ther Res Clin Exp. 2019;91:5-9; doi: 10.1016/j.curtheres.2019.06.001. https://www.ncbi.nlm.nih.gov/pubmed/31372189.

9. Buchter V, Ong YC, Mouvet F, Ladaycia A, Lepeltier E, Rothlisberger U, et al. Multidisciplinary Preclinical Investigations on Three Oxamniquine Analogues as New Drug Candidates for Schistosomiasis*. Chemistry. 2020;26 66:15232-41; doi: 10.1002/chem.202002856. https://www.ncbi.nlm.nih.gov/pubmed/32852116.

10. Buchter V, Hess J, Gasser G, Keiser J. Assessment of tegumental damage to Schistosoma mansoni and S. haematobium after in vitro exposure to ferrocenyl, ruthenocenyl and benzyl derivatives of oxamniquine using scanning electron microscopy. Parasites \& vectors. 2018;11 1:580; doi: 10.1186/s13071-018-3132-x. https://www.ncbi.nlm.nih.gov/pubmed/30400935.

11. Hess J, Panic G, Patra M, Mastrobuoni L, Spingler B, Roy S, et al. Ferrocenyl, Ruthenocenyl, and Benzyl Oxamniquine Derivatives with Cross-Species Activity against Schistosoma mansoni and Schistosoma haematobium. ACS Infect Dis. 2017;3 9:645-52; doi: 10.1021/acsinfecdis.7b00054. https://www.ncbi.nlm.nih.gov/pubmed/28686009.

12. Strohmayer J, Matthews I, Locke R. Schistosomiasis: Traverers in Africa. J Spec Oper Med. 2016;16 3:47-52. https://www.ncbi.nlm.nih.gov/pubmed/27734442.

13. Hotez PJ, Bottazzi ME, Bethony J, Diemert DD. Advancing the Development of a Human Schistosomiasis Vaccine. Trends in parasitology. 2019;35 2:104-8; doi: 10.1016/j.pt.2018.10.005. https://www.ncbi.nlm.nih.gov/pubmed/30455112.

14. Molehin AJ. Schistosomiasis vaccine development: update on human clinical trials. J Biomed Sci. 2020;27 1:28; doi: 10.1186/s12929-020-0621-y. https://www.ncbi.nlm.nih.gov/pubmed/31969170.

15. McCreesh N, Booth $M$. The effect of simulating different intermediate host snail species on the link between water temperature and schistosomiasis risk. PloS one. 2014;9 7:e87892; doi: 10.1371/journal.pone.0087892. https://www.ncbi.nlm.nih.gov/pubmed/24988377.

16. Lardans V, Dissous C. Snail control strategies for reduction of schistosomiasis transmission. Parasitol Today. 1998;14 10:413-7; doi: 10.1016/s0169-4758(98)01320-9. https://www.ncbi.nlm.nih.gov/pubmed/17040832.

17. Adema CM, Hillier LW, Jones CS, Loker ES, Knight M, Minx P, et al. Whole genome analysis of a schistosomiasis-transmitting freshwater snail. Nature communications. 2017;8:15451; doi: 10.1038/ncomms15451. https://www.ncbi.nlm.nih.gov/pubmed/28508897.

18. Matsumura K, Mitsui Y, Sato K, Sakamoto M, Aoki Y. Schistosoma mansoni: possible involvement of protein kinase $\mathrm{C}$ in linoleic acid-induced proteolytic enzyme release from cercariae. Exp Parasitol. 1991;72 3:311-20; doi: 10.1016/0014-4894(91)90151-I. https://www.ncbi.nlm.nih.gov/pubmed/2015870.

19. Ross AG, Bartley PB, Sleigh AC, Olds GR, Li Y, Williams GM, et al. Schistosomiasis. The New England journal of medicine. 2002;346 16:1212-20; doi: 10.1056/NEJMra012396. https://www.ncbi.nlm.nih.gov/pubmed/11961151. 
20. Bahia D, Avelar L, Mortara RA, Khayath N, Yan Y, Noel C, et al. SmPKC1, a new protein kinase C identified in the platyhelminth parasite Schistosoma mansoni. Biochemical and biophysical research communications. 2006;345 3:1138-48; doi: 10.1016/j.bbrc.2006.05.025. https://www.ncbi.nlm.nih.gov/pubmed/16713993.

21. Parker-Manuel SJ, Ivens AC, Dillon GP, Wilson RA. Gene expression patterns in larval Schistosoma mansoni associated with infection of the mammalian host. PLoS neglected tropical diseases. 2011;5 8:e1274; doi: 10.1371/journal.pntd.0001274. https://www.ncbi.nlm.nih.gov/pubmed/21912711.

22. Sullivan JT. Comparison of NMRI Schistosoma mansoni Infection in Three Susceptible Laboratory Albino Strains of Biomphalaria glabrata. J Parasitol. 2019;105 4:576-9. https://www.ncbi.nlm.nih.gov/pubmed/31414948.

23. Agner AE, Granath WO, Jr. Hemocytes of schistosome-resistant and -susceptible Biomphalaria glabrata recognize different antigens on the surface of Schistosoma mansoni sporocysts. J Parasitol. 1995;81 2:179-86. https://www.ncbi.nlm.nih.gov/pubmed/7707192.

24. Ittiprasert W, Nene R, Miller A, Raghavan N, Lewis F, Hodgson J, et al. Schistosoma mansoni infection of juvenile Biomphalaria glabrata induces a differential stress response between resistant and susceptible snails. Exp Parasitol. 2009;123 3:203-11; doi: 10.1016/j.exppara.2009.07.015. https://www.ncbi.nlm.nih.gov/pubmed/19660454.

25. Ittiprasert W, Miller A, Myers J, Nene V, El-Sayed NM, Knight M. Identification of immediate response genes dominantly expressed in juvenile resistant and susceptible Biomphalaria glabrata snails upon exposure to Schistosoma mansoni. Mol Biochem Parasitol. 2010;169 1:27-39; doi: 10.1016/j.molbiopara.2009.09.009. https://www.ncbi.nlm.nih.gov/pubmed/19815034.

26. Castillo MG, Humphries JE, Mourao MM, Marquez J, Gonzalez A, Montelongo CE. Biomphalaria glabrata immunity: Post-genome advances. Dev Comp Immunol. 2020;104:103557; doi: 10.1016/j.dci.2019.103557. https://www.ncbi.nlm.nih.gov/pubmed/31759924.

27. Glinski Z, Jarosz J. Molluscan immune defenses. Archivum immunologiae et therapiae experimentalis. 1997;45 2-3:149-55. https://www.ncbi.nlm.nih.gov/pubmed/9597080.

28. Loker ES, Bayne CJ, Buckley PM, Kruse KT. Ultrastructure of encapsulation of Schistosoma mansoni mother sporocysts by hemocytes of juveniles of the 10-R2 strain of Biomphalaria glabrata. J Parasitol. 1982;68 1:84-94. https://www.ncbi.nlm.nih.gov/pubmed/7077450.

29. Hahn UK, Bender RC, Bayne CJ. Killing of Schistosoma mansoni sporocysts by hemocytes from resistant Biomphalaria glabrata: role of reactive oxygen species. J Parasitol. 2001;87 2:292-9; doi: 10.1645/0022-3395(2001)087[0292:KOSMSB]2.0.CO;2. https://www.ncbi.nlm.nih.gov/pubmed/11318558.

30. Lockyer AE, Spinks J, Kane RA, Hoffmann KF, Fitzpatrick JM, Rollinson D, et al. Biomphalaria glabrata transcriptome: cDNA microarray profiling identifies resistant- and susceptible-specific gene expression in haemocytes from snail strains exposed to Schistosoma mansoni. BMC genomics. 2008;9:634; doi: 10.1186/1471-2164-9-634. https://www.ncbi.nlm.nih.gov/pubmed/19114004. 
31. Ye Q, Dong HF, Grevelding CG, Hu M. In vitro cultivation of Schistosoma japonicum-parasites and cells. Biotechnology advances. 2013;31 8:1722-37; doi: 10.1016/j.biotechadv.2013.09.003. https://www.ncbi.nlm.nih.gov/pubmed/24070875.

32. Voge M, Seidel JS. Transformation in vitro of miracidia of Schistosoma mansoni and S. japonicum into young sporocysts. J Parasitol. 1972;58 4:699-704.

https://www.ncbi.nlm.nih.gov/pubmed/5066549.

33. EL. H. A cell line from embryos of Biomphalaria glabrata (Pulmonata): Establishment and characteristics. In: Maramorosch K, editor In Invertebrate Tissue Culture: Research Applications New York: Academic Press. 1976:75-97.

34. Yoshino TP, Laursen JR. Production of Schistosoma mansoni daughter sporocysts from mother sporocysts maintained in synxenic culture with Biomphalaria glabrata embryonic (Bge) cells. J Parasitol. 1995;81 5:714-22. https://www.ncbi.nlm.nih.gov/pubmed/7472861.

35. Ivanchenko MG, Lerner JP, McCormick RS, Toumadje A, Allen B, Fischer K, et al. Continuous in vitro propagation and differentiation of cultures of the intramolluscan stages of the human parasite Schistosoma mansoni. Proceedings of the National Academy of Sciences of the United States of America. 1999;96 9:4965-70; doi: 10.1073/pnas.96.9.4965.

https://www.ncbi.nlm.nih.gov/pubmed/10220402.

\section{Figures}

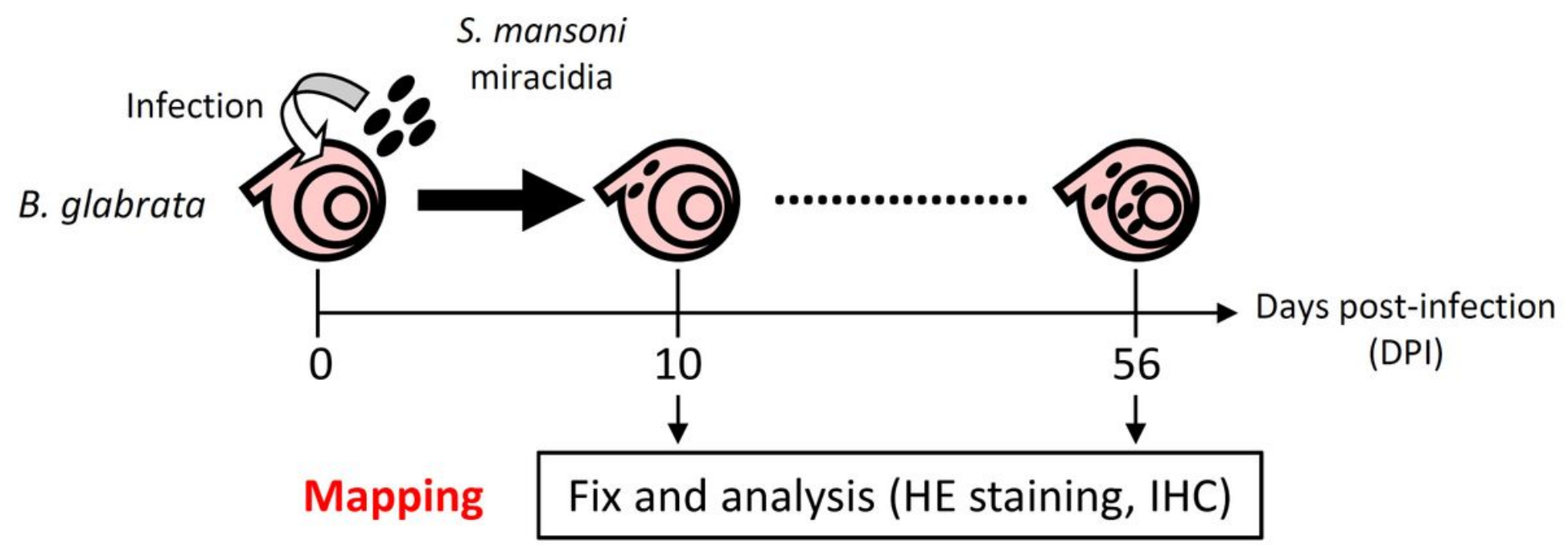

\section{Figure 1}

Study schema Snails infected by S. mansoni miracidia were harvested at 10 and 56 DPI. Sections of fixed snails were analyzed by H\&E staining and immunohistochemistry using anti-S. mansoni antibodies. 

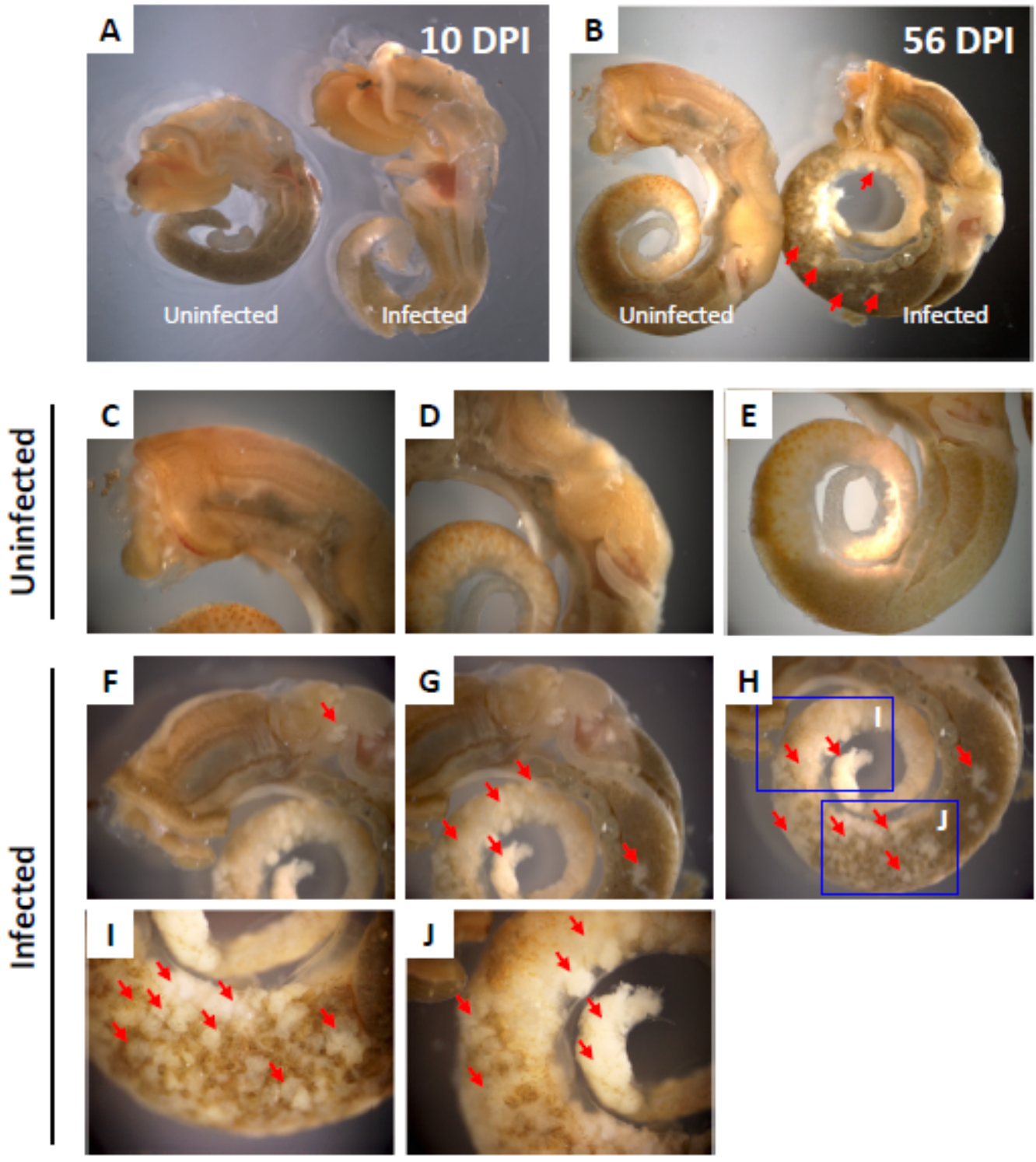

Figure 2

Stereoscopic observations of B. glabrata uninfected or infected with S. mansoni Photographs of B. glabrata snails without and with infection by S. mansoni miracidia at 10 DPI (A) and 56 DPI (B). (C-J) Photographs show B. glabrata snails uninfected (C-E) or infected (F-J) with S. mansoni at 56 DPI. $(I, J)$ Magnification of blue squares shown in $\mathrm{H}$. White mass spots were noted (red arrows). 

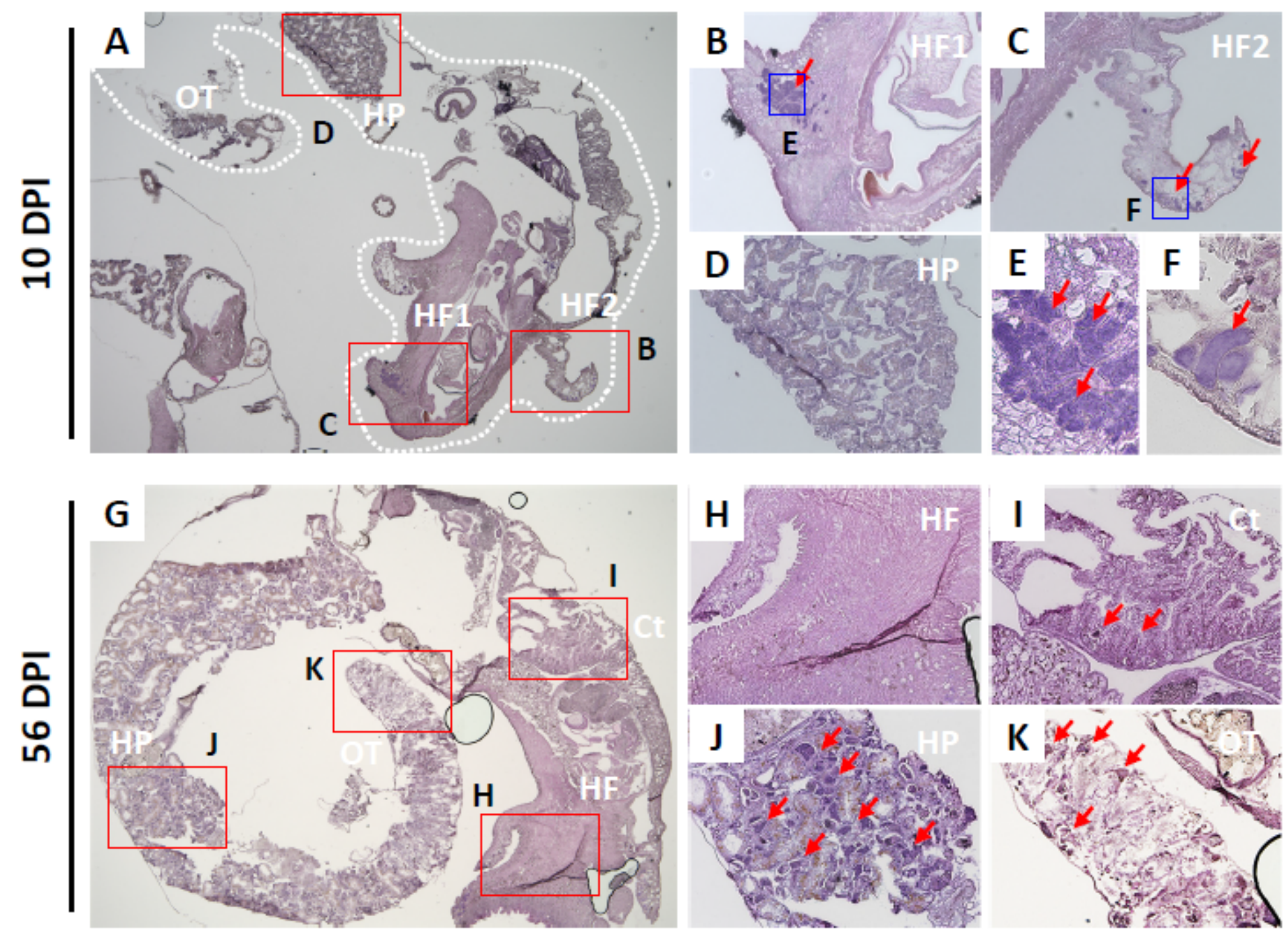

\section{Figure 3}

Histological analysis of S. mansoni larvae in infected-B. glabrata snails (A-F) Photographs show H\&E stained section of B. glabrata snails infected with S. mansoni at 10 DPI. HF1, HF2: head-foot; HP: hepatopancreas; OT: ovotestis. (B-D) Magnification of red squares in A. Arrows indicate S. mansoni larvae infection. $(E, F)$ Magnification of blue squares in $B$ and $C$, respectively. (G-K) Photographs show H\&E stained section of B. glabrata snails infected with S. mansoni at 56 DPI. Ct: ctenidium; HF: headfoot; HP: hepatopancreas; OT: ovotestis. (H-K) Magnification of red squares in G. Arrows indicate S. mansoni larvae infection. 


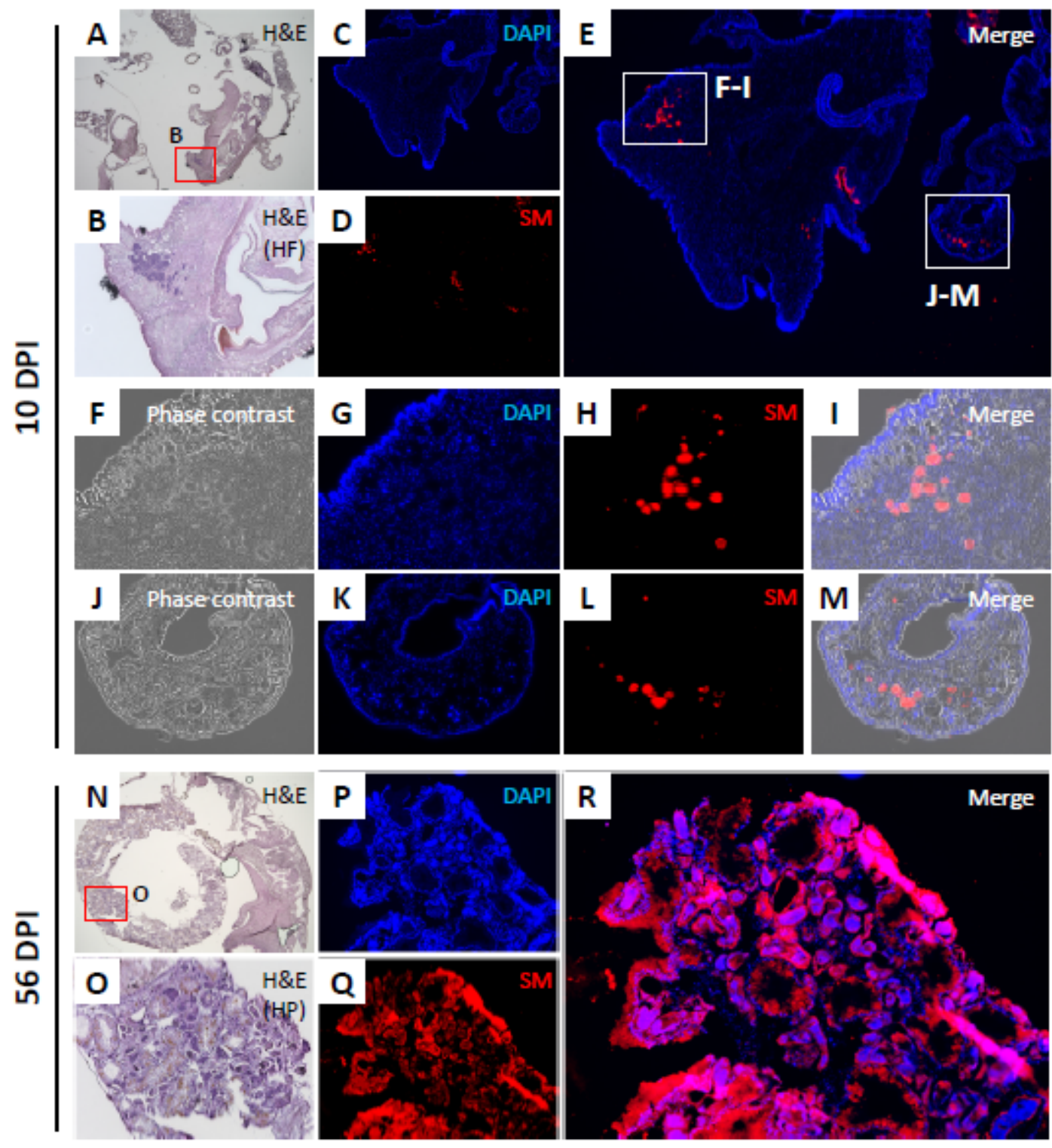

Figure 4

Immunohistochemical analysis of S. mansoni infected with B.glabrata. Sections of infected snails were stained with H\&E (A, B: 10 DPI; N, O: 56 DPI). Figures A, B, N and O in Figure 4 are same respectively to Figures $\mathrm{A}, \mathrm{B}, \mathrm{G}$ and $\mathrm{J}$ in Figure 3 . Areas in red squares in $\mathrm{A}$ and $\mathrm{N}$ are shown magnified in $\mathrm{B}$ and $\mathrm{O}$, respectively. Sections of infected snails were analyzed by immunohistochemistry using anti-S. mansoni antibodies (SM) (C-M: 10 DPI, P-R: 56 DPI). (F-I, J-M) Magnification of squares in E. F, J: phase contrast. C, G, K, P: staining with DAPI. D, H, L, Q: S. mansoni detected immunohistochemically using SM and AlexaFluor 546 conjugated secondary antibodies. E, I, M, R: Merged images with DAPI and SM staining. 
A
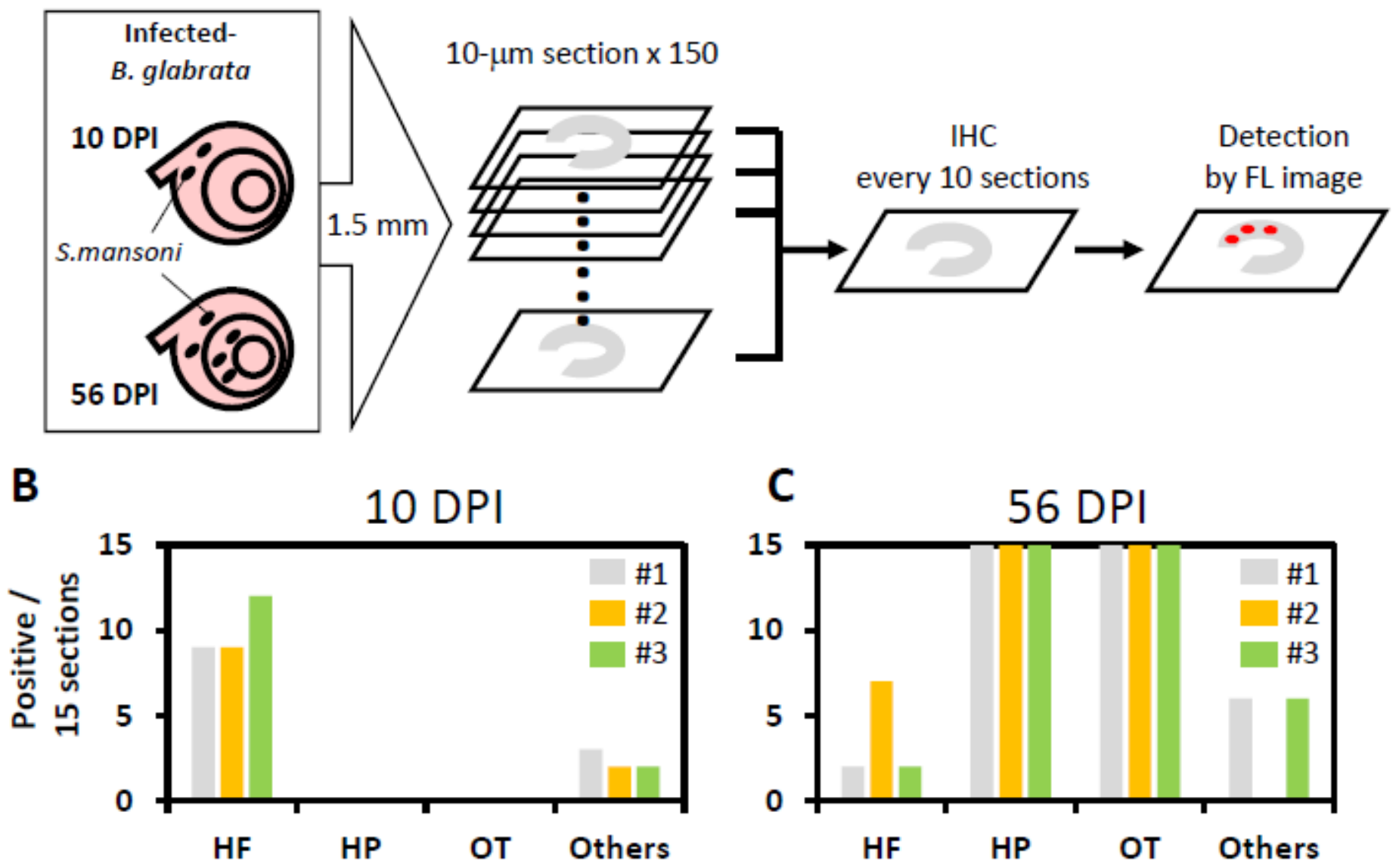

Figure 5

Mapping of S. mansoni larvae in B. glabrata snails and extract preparation. (A) Immunohistochemical analyses were performed using serial sections of snails infected with S. mansoni. (B) At 10 DPI, S. mansoni larvae were predominantly detected in the head-foot (HF) area, though also observed in others such as the heart ( $\mathrm{Hr}$ ) and ctenidium (Ct). (C) At 56 DPI, immunopositivity for S. mansoni larvae was locally concentrated in the hepatopancreas (HP) and ovotestis (OT) areas, which was noted in all of the 15 successive specimens examined. A few S. mansoni larvae were also observed in other organs (HF, Ct).

\section{Supplementary Files}

This is a list of supplementary files associated with this preprint. Click to download.

- SupplFig.pdf 